\title{
Relações entre Eventos Estressores Precoces, personalidade e sintomas psiquiátricos: um estudo exploratório em amostra não clínica
}

\author{
Ismael Ferreira da Costa ${ }^{1}{ }^{1}$ \\ Maria Paula Bento Tomaz $\mathbb{1}^{1}$ \\ Mirelly Gomes de Araújo ${ }^{1}{ }^{1}$ \\ Natany de Souza Batista Medeiros $\oplus^{2}$ \\ Melyssa Kellyane Cavalcanti Galdino ${ }^{1}{ }^{1}$ \\ ${ }^{1}$ Universidade Federal da Paraíba, PB, Brasil. \\ ${ }^{2}$ Universidade Federal do Rio Grande do Norte, RN, Brasil.
}

\begin{abstract}
Resumo
A ocorrência de Eventos Estressores Precoces (EEPs) pode ser um fator determinante para o desenvolvimento de traços de personalidade disfuncionais e transtornos mentais. O objetivo do presente estudo foi analisar a possível relação entre EEPs, características de personalidade e sintomas psiquiátricos em uma amostra não clínica. Trata-se de uma investigação com delineamento transversal, onde foram utilizados para coleta de dados um questionário sociodemográfico, o Inventário de Autoavaliação para Adultos (ASRI), o Questionário sobre Traumas na Infância (QUESI) e o Inventário de Temperamento e Caráter (ITC-R). Os resultados expõem associação entre a ocorrência de EEPs e maior presença do fator de temperamento evitação de danos e menor presença do fator de caráter autodirecionamento, ambos também apresentaram correlações com sintomas psiquiátricos. Sugere-se que EEPs possam exercer influência sobre o desenvolvimento de traços de personalidade que funcionem como fatores de vulnerabilidade, contribuindo para o surgimento de sintomas psiquiátricos.
\end{abstract}

Palavras-chave: trauma infantil, temperamento, caráter.

\section{Relations between early life stress, temperament and character and psychiatric symptoms: an exploratory study in a non-clinical sample}

\begin{abstract}
The occurrence of Early Life Stress (ELSs) can be a determining factor for the development of dysfunctional personality traits and mental disorders. The aim of this study was to analyze the possible relationship between ELSs, personality traits and psychiatric symptoms in a non clinical sample. It is an investigation with a cross-sectional design, where they were used for data collection: a sociodemographic questionnaire, the Inventory Self-Assessment for Adults (ASI), the Questionnaire on Trauma in Childhood (CTQ) and the Inventory of Temperament and Character (CTI-R). The results expose association between the occurrence of ELSs and greater presence temperament factor harm avoidance and minor character factor presence self-directedness, both also showed correlations with psychiatric symptoms. It is suggested that ELSs may influence the development of personality traits that serve as vulnerability factors for the emergence of psychiatric symptoms.
\end{abstract}

Keywords: childhood trauma, temperament, character.

\section{Relaciones entre eventos estresantes prematuros, personalidad y síntomas psiquiátricos: un estudio exploratorio en una muestra no clínica}

\section{Resumen}

La ocurrencia de Eventos Estresantes Prematuros (EEPs) puede ser un factor determinante para el desarrollo de características de personalidad disfuncional y trastornos mentales. El objetivo del presente estudio fue analizar la posible relación entre EEPs, características de personalidad y síntomas psiquiátricos en una muestra no clínica. Se trata de una investigación con delineamiento transversal, para la recolección de datos fueron utilizados: un cuestionario socio-demográfico, el Inventario de Auto Evaluación para Adultos (ASI), el Cuestionario sobre Traumas en la Infancia (CTQ) y el Inventario de Temperamento y Carácter (ITC). Los resultados exponen una asociación entre la ocurrencia de Estrés EEPs y la mayor presencia del factor de temperamento prevención de daños y la menor presencia del factor carácter Auto direcionamiento, ambos también presentaron correlaciones con síntomas psiquiátricos. Se sugiere que el EEPs pueda ejercer influencia sobre el desenvolvimiento de características de la personalidad que funcionen como factores de vulnerabilidad, contribuyendo para el surgimiento de síntomas psiquiátricos.

Palabras clave: trauma infantil, temperamento, carácter. 
As relações entre a presença de Eventos Estressores Precoces (EEPs) ou traumas na infância e adolescência e o desenvolvimento de transtornos mentais na vida adulta têm se mostrado um campo de investigação esclarecedor nos últimos anos (Teicher \& Samson, 2016). Diversos estudos têm exposto de forma clara o impacto que adversidades durante tais períodos podem exercer sobre o estabelecimento de vulnerabilidades específicas ao desenvolvimento de transtornos mentais (Carr, Martins, Stingel, Lemgruber, \& Juruena, 2013).

Os EEPs podem assumir diversos formatos, entretanto, alguns são mais amplamente investigados em comparação a outros, a exemplo dos abusos físico, sexual, emocional, e das negligências física e emocional (Martins, Tofoli, Baes, \& Juruena, 2011; Kalmakis \& Chandler, 2014). Esses tipos de EEPs são descritos como apresentando associação com transtornos mentais diversos, como depressão (Martins, Von Werne Baes, Tofoli \& Juruena, 2014), transtorno bipolar (Leverich $\&$ Post, 2006), transtornos de ansiedade (Hovens et al., 2012), esquizofrenia (Sahin et al., 2013), abuso de substâncias (Banducci, Hoffman, Lejuez, \& Koenen, 2014), transtornos de personalidade (Zhang, Chow, Wang, Dai, \& Xiao, 2012) e risco de suicídio (Barbosa et al., 2014).

Muitos estudos têm buscado justificar esta associação através do entendimento dos efeitos do estresse sobre o cérebro durante a infância e a adolescência. A ocorrência de EEPs nestes períodos pode alterar de forma permanente o padrão de funcionamento do Eixo Hipotalâmico Hipofisário Adrenal (Hipotalamic Ptuitary Adrenal-HPA), sistema responsável pela regulação dos mecanismos neuroendócrinos relacionados ao estresse (Lupien, McEwen, Gunnar \& Heim, 2009; Frodl \& O'Keane, 2013). Essas mudanças levam a um aumento na liberação de glicocorticóides e mineralocorticóides no organismo, tendo como consequência um impacto sobre o desenvolvimento de estruturas cerebrais que possuem receptores para esses hormônios (a exemplo do córtex pré-frontal, hipocampo e amígdala) e exercem papel de feedback negativo, regulando o funcionamento do HPA (Teicher \& Samson, 2016).

Apesar de haver significativo consenso no que diz respeito aos efeitos dos EEPs sobre o funcionamento do eixo HPA e o desenvolvimento de diversas estruturas cerebrais cruciais à regulação emocional e aos comportamentos adaptativos frente a eventos estressores, ainda não existe compreensão similar acerca do possível impacto que os EEPs podem exercer sobre outros elementos centrais ao funcionamento humano saudável, a exemplo da personalidade.

Diversos estudos têm apontado que a presença de traços específicos de personalidade podem funcionar como fatores de vulnerabilidade importantes ao desenvolvimento de transtornos mentais diversos, como depressão, transtorno bipolar, esquizofrenia, abuso de substância, transtornos alimentares, fobia social, transtorno de pânico, transtorno de estresse pós-traumático, transtorno de ansiedade generalizada (Fassino, Amianto, Sobrero, \& Abbate Daga, 2013) e transtornos de personalidade (Wright \& Simms, 2015).

Sobre a relação entre trauma na infância e características de personalidade, observa-se que a presença de EEPs está positivamente associada a características de personalidade como neuroticismo, abertura à experiência, extroversão e psicoticismo, e negativamente associados à autodirecionamento e cooperatividade (Hengartner et al., 2014; De Carvalho et al., 2014; Li et al., 2014). Apesar da relação entre EEPs e características de personalidade ser evidenciada, poucos estudos buscam relacionar estes dois elementos a sintomas psiquiátricos ao mesmo tempo, e, sobretudo, levando em consideração um modelo de personalidade que se proponha a investigar tanto características de personalidade mais flexíveis à influência ambiental, como características mais estáveis e menos reativas ao ambiente.

Um modelo de personalidade que defende esta compreensão é o modelo psicobiológico de Cloninger (Cloninger, Svrakic, \& Svrakic, 1997; Cloninger, Svrakic, \& Przybeck, 1993). Nele postula-se que a personalidade é composta por temperamento e caráter, dois domínios que interagem regulando o desenvolvimento das características de personalidade das pessoas.

O temperamento compõe o domínio predominantemente hereditário da personalidade, caracterizado pelos traços que se manifestam desde o início da vida, de forma mais independente do meio externo. Reflete as respostas automáticas do indivíduo a estímulos emocionalmente relevantes, incluindo respostas emocionais básicas como medo, raiva e apego. São propostos quatro fatores de temperamento: evitação de danos, busca de novidades, dependência de recompensa e persistência. O caráter envolve as diferenças individuais dos processos cognitivos superiores e valores desenvolvidos pela pessoa durante sua vida através de suas experiências. Diferente do temperamento, o caráter é mais flexível à mudança com a idade e à maturação, sendo influenciado por fatores como os já existentes traços de temperamento, as pressões socioculturais e as experiências de vida únicas do indivíduo. São descritos três fatores de caráter: autodirecionamento, cooperatividade e autotranscendência (Svrakic et al., 2002). 
Svrakic \& Cloninger (2010) sugerem que o desenvolvimento da personalidade reflete um complexo esforço adaptativo voltado à auto-organização do indivíduo objetivando um adequado ajuste entre suas necessidades internas (temperamento emocional) e demandas externas (experiências ambientais, normas e costumes sociais). Essa adaptação pode ocorrer de maneira disfuncional, devido à presença de disposições comportamentais prévias extremas e inflexíveis, por efeitos de um ambiente desadaptativo, ou por ambos.

Levando em consideração a influência que o ambiente exerce sobre a personalidade (Cloninger et al., 1993) e a importância da infầncia nesse sentido (Josefsson et al., 2013), sugere-se que eventos traumáticos ocorridos na infância podem influenciar no desenvolvimento de traços de personalidade. Caso esse ambiente tenha sido nocivo durante a infância, acredita-se que haja possibilidade de que essas características se apresentem distintas na vida adulta em comparação a pessoas sem esse histórico. Neste sentido, resultados de alguns estudos apontam que adultos com histórico de EEPs apresentam médias mais altas no fator de temperamento evitação de danos e médias mais baixas no fator de caráter autodirecionamento em comparação com adultos sem esse histórico, ambas as características que podem levar a vulnerabilidades para o desenvolvimento de sintomas psiquiátricos (De Clercq, Rettew, Althoff \& De Bolle, 2012; De Carvalho et al., 2014).

Com base nesta compreensão, o objetivo do presente estudo é identificar as possíveis relações entre EEPs, características de temperamento e caráter e sintomas psiquiátricos em uma amostra não clínica. Sugere-se que histórico de EEPs estará relacionado com a presença de características específicas de personalidade e sintomas psiquiátricos, e que alguns fatores da personalidade também apresentarão associação com sintomas psiquiátricos.

\section{Método}

\section{Desenho do estudo}

O presente estudo caracteriza-se como de natureza exploratória, descritiva, correlacional, transversal e quantitativa.

\section{Participantes}

A amostra do presente estudo foi obtida de forma não probabilística com a utilização do método bola de neve. No total, a amostra foi composta por 200 pessoas de ambos os sexos, apresentando entre 18 e 30 anos de idade e alfabetizadas, oriundas das cidades de João Pessoa (PB), Mari (PB) e Porteiras (CE).

\section{Instrumentos}

\section{Questionário sociodemográfico}

Instrumento desenvolvido pelos pesquisadores, composto por 21 itens e voltado à coleta de informações gerais da amostra, como idade, sexo, religião, estado civil, escolaridade, situação laboral e renda familiar.

\section{Inventário de Autoavaliação para Adultos (ASRI)}

Instrumento voltado à avaliação quantitativa de sintomas psiquiátricos de 19 transtornos mentais, conforme proposto pelo DSM-IV, desenvolvido por Gadow, Sprafkin \& Weiss (2004). É composto por 136 itens que devem ser classificados em escala Likert que varia de 0 (nunca) a 3 (muito frequentemente). Para o presente estudo, foram utilizadas as escalas para os seguintes transtornos: Transtorno de Ansiedade Generalizada (TAG), Transtorno de Pânico (TP), Transtorno Obsessivo Compulsivo (TOC), Fobia Social, Transtorno de Estresse Pós-Traumático (TEPT), Transtorno Depressivo Maior (TDM), Mania, Distimia, Transtorno por Uso de Substância (TUS), Esquizofrenia, Transtorno de Personalidade Esquizoide e Transtorno de Personalidade Borderline. $\mathrm{O}$ instrumento produz dois escores, um para cada transtorno, através de ponto de corte que aponta a presença ou não de sintomas baseado nos critérios estabelecidos pelo DSM-IV, o outro escore é baseado na severidade dos sintomas, que mensura a sintomatologia total em cada transtorno. Este último possibilita, ainda, a geração de um escore final total de sintomas a partir da soma das escalas de todos os transtornos. Não existem dados brasileiros de adaptação cultural ou validação para este instrumento. Por esse motivo, foi utilizada a tradução para o português da versão original realizada pelos pesquisadores que se encontra em processo de validação. Análises inicias do ASRI apresentam boas características psicométricas (alpha de Cronbach de 0.96).

\section{Questionário sobre Traumas na Infância (QUESI)}

Entrevista retrospectiva e autoaplicável desenvolvida por Bernstein et al. (2003) que investiga história de abuso e negligência durante a infância, podendo ser aplicada para adolescentes (a partir de 12 anos) e adultos, composta por 28 itens organizados em uma escala Likert que varia de 1 (nunca) a 5 (muito frequentemente). $\mathrm{O}$ instrumento avalia cinco subtipos de traumas na infância: abuso físico, abuso emocional, abuso sexual, negligência física e negligência emocional. As pontuações variam de 5 a 25 para cada tipo de abuso. 
O grau de severidade de cada abuso é categorizado em quatro níveis. Indivíduos que apresentam escores 'moderado a severo' ou 'severo a extremo' em qualquer subtipo de trauma, são identificados com histórico de EEPs. Já os categorizados entre os escores 'nenhum a mínimo' e 'leve a moderado' são considerados sem histórico de EEPs. O instrumento ainda permite a produção de um escore total a partir do somatório dos escores de todos os cinco subtipos de EEPs. O QUESI foi traduzido e adaptado à população brasileira (Grassi-Oliveira, Stein, \& Pezzi, 2006) e apresenta boas características psicométricas (Grassi-Oliveira et al., 2014).

\section{Inventário de Temperamento e Caráter-Revisado (ITC-R)}

Questionário desenvolvido por Cloninger (1987) e revisado por Cloninger et al. (1993) voltado à avaliação de características de temperamento e caráter. Abrange quatro fatores relacionados ao temperamento (evitação de danos, busca de novidade, dependência de recompensa e persistência) e três fatores relacionados ao caráter (autodirecionamento, cooperatividade e autotranscendência). O ITC possui 240 itens que são classificados de acordo com uma escala Likert que varia de 1 (definitivamente falso) a 5 (definitivamente verdadeiro). $\mathrm{O}$ instrumento produz escores totais $\mathrm{e}$ médios para cada fator de temperamento e caráter avaliado. Para o presente estudo foram utilizados os valores totais. O inventário encontra-se validado e normatizado para ser utilizado na população brasileira apresentando boas propriedades psicométricas (Goncalves \& Cloninger, 2010).

\section{Procedimentos}

\section{Coleta de dados}

Os dados foram coletados de forma individual. Tendo em vista a extensão do protocolo e a possibilidade do efeito da fadiga durante o seu preenchimento, a coleta de dados ocorreu em duas etapas. Na primeira, após assinatura do Termo de Consentimento Livre e Esclarecido (TCLE), os participantes responderam o questionário sociodemográfico e o Inventário de Autoavaliação para Adulto (ASRI). Em média, esta primeira etapa durou 30 minutos. Na segunda etapa foram respondidos o Questionário sobre Traumas na Infância (QUESI) e o Inventário de Temperamento e Caráter-Revisado (ITC-R), esta aplicação teve duração média de 40 minutos. Antes do início da aplicação, todos os instrumentos referentes a cada etapa eram apresentados e a forma de se responder era devidamente esclarecida. O intervalo entre a aplicação das duas etapas obedeceu a um período máximo de 10 dias.

\section{Análise de dados}

Os dados foram tabulados com o auxílio do programa Statistical Package for the Social Sciences (SPSS versão 20.0; 2011). As análises descritivas foram realizadas tendo por base o estabelecimento de médias, desvio-padrão, frequências e porcentagem. Para as análises inferenciais de comparação de dois grupos foram utilizados o teste $t$ de student. Para as análises de correlação foi utilizado o teste de Pearson. Para todos os dados analisados foram consideradas significativas as diferenças com valores $\mathrm{p}<0.05$.

\section{Considerações éticas}

Este estudo foi submetido e aprovado pelo Comitê de Ética e Pesquisa (CEP) do Centro de Ciências da Saúde (CCS) da Universidade Federal da Paraíba (UFPB) (Protocolo 0141/15. CAEE: 43221215.6.00005188). Desta forma, toda a pesquisa foi realizada conforme a Resolução no 466/12 do Conselho Nacional de Saúde (CNS) do Ministério da Saúde (MS), que trata das diretrizes e normas de pesquisas que envolvem seres humanos.

\section{Resultados}

\section{Caracterização da amostra de acordo com os dados sociodemográficos}

A amostra total do presente estudo foi composta predominantemente por participantes do sexo feminino $(n=170,65 \%)$, de cor parda $(n=88,44 \%)$; solteiros (as) $(n=171,85.5 \%)$ e estudantes $(n=155)$ cursando o ensino superior $(n=124,63.3 \%)$. Com relação à religião, a maioria se descreveu como católica $(n=95$, $47.7 \%)$ e morando com os seus pais $(n=136,68.3 \%)$. A idade média da amostra geral foi de 22,70 ( $D P=3.52)$. Para análise comparativa das variáveis em estudo, a amostra foi dividida em dois grupos, um apresentado como 'Com histórico de EEPs' e outro descrito como 'Sem histórico de EEPs'. A amostra descrita com EEPs foi formada por 100 participantes $(50 \%)$ e a sem histórico de estresse precoce também com 100 participantes $(50 \%)$. Dentre os participantes que foram identificados com histórico de EEPs, 54 (27\%) sofreram abuso emocional; 39 (19.5\%) negligência emocional, $38(19 \%)$ abuso físico; 29 (14.5\%) negligência física e $18(9.6 \%)$ abuso sexual.

\section{Caracterização da amostra de acordo com EEPs e sintomas psiquiátricos}

Como apresentado pela Tabela 1, a presença de sintomatologia psiquiátrica é significativamente maior no grupo caracterizado com EEPs tanto com respeito 
à totalidade dos sintomas psiquiátricos $(t(193)=5.79$, $p<0.01$ ), como também em cada transtorno avaliado, dentre eles: transtorno de ansiedade generalizada $(t(198)=4.30, \quad p<0.01)$; depressão $(t(198)=5.16$, $p<0.01)$; fobia social $(t(198)=3.18, p<0.05)$; distimia $(t(198)=4.38, p<0.01)$ e transtorno de personalidade borderline $(t(197)=4.88, p<0.01)$.

As correlações entre o escore total de EEPs e os sintomas psiquiátricos expõem associação positiva de moderada intensidade $(r=0.46, p<0.01)$, a mesma tendência é observada com os outros subtipos de EEPs, com exceção do abuso físico $(r=0.14, p<0.05)$, onde a correlação não foi significativa. Entre sintomas psiquiátricos e abuso emocional também houve correlação positiva moderada $(r=0.47, p<0.01)$. Apesar das intensidades menores, os sintomas psiquiátricos também apresentaram correlação com negligência emocional $(r=0.35, p<0.01)$, negligência física $(r=0.28$, $p<0.01)$ e abuso sexual $(r=0.27, p<0.01)$.

\section{Caracterização da amostra de acordo com fatores de temperamento e caráter e EEPs}

Com relação às médias referentes aos fatores de temperamento e ao caráter entre os grupos com e sem histórico de EEPs, o único fator que apresentou diferenças significativas foi autodirecionamento da dimensão caráter $(t(181)=4.34, p<0.01)$, com média significativamente inferior no grupo com EEPs. Os demais fatores não apresentaram diferenças significativas entre os dois grupos.

Nas correlações apresentadas na Tabela 2, entre temperamento e EEPs, dois fatores desta dimensão apresentaram correlações fracas, evitação de danos correlacionou-se de forma positiva $(r=0.20, p<0.01)$ e dependência de recompensa de forma negativa $(r=0.16$, $p<0.01)$ com o escore total deEEPs. Na dimensão caráter, os fatores autodirecionamento $(r=-0.39, p<0.01) \mathrm{e}$ cooperatividade $(r=-0.15, p<0.05)$ apresentaram ambas correlações fracas e negativas com EEPs. Com relação aos subtipos de EEPs, negligência e abuso emocional e negligência física foram os que apresentaram maior número de correlações com os fatores de temperamento e caráter. Abuso físico não apresentou correlação com nenhum fator de temperamento ou caráter. Dentre todos os fatores, o que apresentou maior quantidade de correlações foi autodirecionamento. A correlação mais representativa desse fator foi moderada e negativa com abuso emocional $(r=-0.40, p<0.01)$.

TABELA 1

Distribuição da sintomatologia psiquiátrica entre os grupos com e sem histórico de EEPs

\begin{tabular}{|c|c|c|}
\hline Transtornos & $\begin{array}{c}\text { Sem Estresse Precoce } \\
M(D P)\end{array}$ & $\begin{array}{c}\text { Com Estresse Precoce } \\
M(D P)\end{array}$ \\
\hline Sintomas Psiquiátricos (Total) & $28.06(16.38)$ & $43.22(19.98)^{* * *}$ \\
\hline TAG & $8.03(3.90)$ & $10.61(4.54)^{* *}$ \\
\hline $\mathrm{TP}$ & $1.36(1.34)$ & $1.92(1.50)^{*}$ \\
\hline TOC & $0.84(1.18)$ & $1.96(2.15)^{* *}$ \\
\hline Fobia social & $2.46(2.06)$ & $3.42(2.19)^{*}$ \\
\hline TEPT & $1.43(1.35)$ & $2.17(1.77)^{*}$ \\
\hline TDM & $2.85(2.77)$ & $5.11(3.38)^{* *}$ \\
\hline Distimia & $1.79(1.74)$ & $2.99(2.11)^{* *}$ \\
\hline Tr. P. Borderline & $3.51(3.13)$ & $5.93(3.81)^{* *}$ \\
\hline
\end{tabular}

M: Média; DP: Desvio Padrão; TAG: Transtorno de Ansiedade Generalizada; TP: Transtorno de Pânico; Transtorno Obsessivo Compulsivo; TEPT: Transtorno de Estresse Pós-Traumático; TDM: Transtorno Depressivo Maior; TBH: Tr. P.: Transtorno de Personalidade. * $p<0.05$; ** $p<0.01 ; * * * p<0.001$.

TABELA 2

Correlação entre fatores de temperamento/caráter e total e subtipos de EEPs

\begin{tabular}{|c|c|c|c|c|c|c|}
\hline \multirow{2}{*}{$\begin{array}{l}\text { Fatores de } \\
\text { Temperamento e Caráter }\end{array}$} & EEPs (Total) & $A E$ & $A F$ & $A S$ & $N E$ & $N F$ \\
\hline & \multicolumn{6}{|c|}{$r$} \\
\hline T1 - Evitação de Danos & $0.20 * *$ & $0.22 * *$ & -0.01 & 0.10 & $-0.21 * *$ & 0.09 \\
\hline T2 - Busca de Novidade & 0.02 & 0.31 & 0.36 & -0.01 & 0.01 & 0.05 \\
\hline T3 - Dependência de Recompensa & $-0.16^{*}$ & 0.49 & 0.05 & -0.05 & $-0.29 * *$ & $-0.19^{*}$ \\
\hline T4 - Persistência & -0.11 & 0.10 & 0.56 & 0.02 & $-0.26^{* *}$ & -0.01 \\
\hline $\mathrm{C} 1$ - Autodirecionamento & $-0.39 * *$ & $-0.40 * *$ & -0.11 & $-0.18^{*}$ & -0.31 & $-0.28 * *$ \\
\hline $\mathrm{C} 2$ - Cooperatividade & $-0.15^{*}$ & -0.07 & 0.38 & -0.01 & $-0.16^{*}$ & $-0.22 * *$ \\
\hline C3 - Autotranscendência & 0.09 & $0.26^{* *}$ & 0.16 & 0.03 & -0.57 & -0.05 \\
\hline
\end{tabular}

AE: Abuso Emocional; AF: Abuso Físico; AS: Abuso Sexual; AE: Negligência Emocional; NF: Negligência Física. * $p<0.05$; $* * p<0.01$. 


\section{Caracterização da amostra de acordo com as dimensões de temperamento e caráter $\mathrm{e}$ sintomas psiquiátricos}

As médias dos fatores de temperamento e caráter de acordo com a presença ou não de sintomas psiquiátricos são apresentadas na Tabela 3 e demonstram diferenças significativas em dois fatores. Evitação de danos, da dimensão de temperamento, apresentou escore médio superior no grupo com sintomas psiquiátricos $(t(179)=4.41, \mathrm{p}<0.01)$ e autodirecionamento apresentou média inferior no grupo com sintomas psiquiátricos $(t(179)=9.80, \mathrm{p}<0.01)$.

$\mathrm{Na}$ Tabela 4 encontram-se as correlações entre os fatores de temperamento e caráter e o escore total de sintomas psiquiátricos. $\mathrm{O}$ fator de temperamento evitação de danos apresenta correlação positiva e moderada com os sintomas psiquiátricos $(r=0.49$, $p<0.01)$. Dentre os demais fatores de temperamento, dependência de recompensa $(r=-0.09, p<0.01)$ e persistência $(r=-0.16, p<0.01)$ apresentam correlação fraca e negativa. Na dimensão caráter, observa-se correlação moderada e negativa entre o fator autodirecionamento e sintomas psiquiátricos $(r=-0.69, p<0.01)$ e correlação fraca e negativa entre o fator cooperatividade e sintomas psiquiátricos $(r=-0.21, p<0.01)$.

\section{Discussão}

A relação entre EEPs e transtornos mentais vem sendo identificada de forma recorrente em muitos estudos (Martins et al., 2011). De forma similar, há grande consenso no que diz respeito aos impactos que esses eventos exercem sobre o sistema neuroendócrino e sua correspondente relação com vulnerabilidade ao desenvolvimento de transtornos mentais (Lupien et al., 2009). Apesar desse entendimento, não se observa correspondente compreensão com relação à associação entre EEPs e o desenvolvimento da personalidade, no sentido de esclarecer se esses eventos estão associados à presença de características de personalidade que atuem como fatores de vulnerabilidade sobre $\mathrm{o}$ desenvolvimento de sintomas psiquiátricos.

Os resultados obtidos neste estudo expõem relações significativas entre EEPs, características de temperamento e caráter, e sintomas psiquiátricos. Os dados sugerem que a presença de EEPs podem exercer influência sobre o desenvolvimento de características de personalidade, que atuariam como fatores de vulnerabilidade importantes sobre o desenvolvimento de sintomas psiquiátricos.

Sobre a incidência comparativa de sintomas psiquiátricos entre os grupos com e sem histórico de

TABELA 3

Distribuição dos fatores de temperamento e caráter de acordo com os subgrupos sem e com sintomas psiquiátricos

\begin{tabular}{lcl}
\hline & $\begin{array}{c}\text { Sem sintomas psiquiátricos } \\
n=149(75.3 \%)\end{array}$ & $\begin{array}{c}\text { Com sintomas psiquiátricos } \\
n=49(24.7 \%)\end{array}$ \\
\cline { 2 - 3 } & Média (DP) & Média (DP) \\
\hline T1 - Evitação de Danos & $60.37(9.10)$ & $68.73(11.70)^{* *}$ \\
T2 - Busca de Novidades & $55.79(6.57)$ & $56.27(6.89)$ \\
T3 - Dependência de Recompensa & $67.70(9.11)$ & $65.91(12.04)$ \\
T4 - Persistência & $64.81(8.83)$ & $61.51(11.71)$ \\
C1 - Autodirecionamento & $72.10(9.32)$ & $58.04(8.05)^{* *}$ \\
C2 - Cooperatividade & $77.19(8.27)$ & $74.22(9.05)$ \\
C3 - Autotranscendência & $57.80(10.56)$ & $57.97(14.30)$ \\
\hline
\end{tabular}

M: Média; DP: Desvio Padrão. ** $p<0.01$.

TABELA 4

Correlação entre fatores de temperamento e caráter e sintomas psiquiátricos

\begin{tabular}{lc}
\hline & Sintomas Psiquiátricos (Total) \\
T1 - Evitação de Danos & $r$ \\
T2 - Busca de Novidades & $0.49^{* *}$ \\
T3 - Dependência de Recompensa & 0.10 \\
T4 - Persistência & $-0.09^{* *}$ \\
C1 - Autodirecionamento & $-0.16^{* *}$ \\
C2 - Cooperatividade & $-0.69^{* *}$ \\
C3 - Autotranscendência & $-0.21^{* *}$ \\
\hline
\end{tabular}

$r$ : Correlação de Pearson. ** $p<0.01$. 
EEPs, observou-se maior presença de sintomatologia psiquiátrica no grupo categorizado com histórico de EEPs (Tabela 1). Além disso, também se identificou correlação positiva e moderada entre os escores totais de sintomas psiquiátricos e EEPs e entre todos os demais subtipos de Estresse Precoce, com exceção de abuso físico. Estes resultados reiteram um dos achados mais amplamente notificados neste campo de estudos, que relaciona a presença de EEPs como estando associada a uma maior vulnerabilidade ao desenvolvimento de sintomas psiquiátricos e transtornos mentais (Kessler et al., 2010; Martins et al., 2011; Carr et al., 2013).

Com relação a presença de EEPs e o fator caráter de personalidade, os resultados apontam correlação negativa entre os fatores autodirecionamento e cooperatividade com EEPs (Tabela 2), resultado que expõe uma associação entre a ocorrência de EEPs e prejuízos no desenvolvimento de características de personalidade que são descritas como fatores de proteção para o desenvolvimento de transtornos mentais (Cloninger et al., 1997; Cloninger et al., 1993). Rademaker, Vermetten, Geuze, Muilwijk, \& Kleber, (2008) e De Carvalho et al., (2014) obtiveram resultados similares, observaram que a presença de traumas na infância estão associados com baixos escores em autodirecionamento e cooperatividade.

No que diz respeito ao temperamento, os resultados obtidos neste estudo apontam não haver distinção em nenhum fator de temperamento entre o grupo com e sem histórico de EEPs. A presença de EEPs aparentemente não interfere de forma significativa no percurso de desenvolvimento destes fatores, dado já esperado, pois teoricamente se defende que os fatores de temperamento são menos reativos às experiência ambientais em comparação com os fatores de caráter (Rademaker et al., 2008). Por outro lado, as correlações expõem associação positiva e moderada entre EEPs e evitação de danos e associação negativa e fraca entre dependência de recompensa e EEPs. Dado similar foi obtido por De Carvalho et al. (2014). Os autores enfatizam que características especificas de temperamento podem em conjunto com a ocorrência de EEPs levarem a uma maior probabilidade de produzirem efeitos prejudiciais às pessoas que os apresentam. Fassino et al. (2013) também descrevem evitação de danos como sendo um fator de vulnerabilidade central para o desenvolvimento de transtornos mentais.

Com relação à distribuição diferenciada das dimensões de temperamento e caráter para os grupos categorizados com e sem sintomas psiquiátricos, observou-se diferenças significativas no fator evitação de danos da dimensão temperamento, com escores mais altos no grupo com sintomas psiquiátricos, e autodirecionamento da dimensão caráter, com escores mais baixos também no grupo com sintomas psiquiátricos (Tabela 3). Nas correlações também se destacam as associações moderadas para ambos os fatores; correlação positiva entre evitação de danos e sintomas psiquiátricos e correlação negativa entre autodirecionamento e sintomas psiquiátricos (Tabela 4). Correlações negativas e fracas também foram identificadas entre os fatores de temperamento dependência de recompensa e persistência com sintomas psiquiátricos, e entre a dimensão de caráter cooperatividade e sintomas psiquiátricos.

Os dados apresentados acima são encontrados em outras investigações. Em revisão de literatura, Fassino et al. (2013), evitação de danos e autodirecionamento foram identificados como fatores de vulnerabilidade centrais para o desenvolvimento, manutenção, severidade e recorrência de transtornos mentais, sendo também um preditor de insucesso em tratamentos psicoterápicos e farmacológicos. Em diversos transtornos categorizados no eixo I do DSM-IV-TR observou-se que evitação de danos apresentou escores significativamente altos em $88 \%$ dos estudos e autodirecionamento apresentou escores significativamente baixos em $96 \%$.

Apesar dos resultados relevantes discutidos neste estudo, alguns elementos não foram investigados e caracterizam limitações. Uma limitação diz respeito à identificação do(s) agressor(es) do abuso/negligência. Sugere-se que quando a figura do abusador é a mãe ou o pai, os potenciais efeitos desses eventos tendem a ser mais graves e permanentes (McCarthy \& Lumley, 2012). Outra limitação identificada refere-se a não observância da frequência como também à multiplicidade de EEPs. Diversos estudos apontam que, quando há recorrência e a pessoa foi vítima de mais de um tipo de trauma, há um efeito cumulativo nos prejuízos decorrentes (Arata, Langhinrichsen-Rohling, Bowers, \& O'Brien, 2007; Finkelhor, Ormrod, \& Turner, 2007).

Os resultados obtidos nesse estudo, Embora com algumas limitações, são indicativos importantes da relação entre EEPs e o desenvolvimento de traços de personalidade desadaptativos, expondo também as associações entre essas características de personalidade com vulnerabilidade ao desenvolvimento de sintomas psiquiátricos. A compreensão do impacto que os EEPs podem trazer sobre as suas vítimas encerra grande importância, a princípio por conscientizar a sociedade sobre os muitos prejuízos que esses eventos podem trazer e, também, por caracterizar conhecimento útil no suporte adequado a pessoas que possuem histórico de EEPs. Salienta-se que pessoas com transtornos mentais que tenham sido vítimas desses eventos, costumeiramente apresentam sintomas psiquiátricos 
e percurso de tratamento diferenciado, marcado por maior gravidade e recorrência quando comparados à população clínica que não apresenta histórico de EEPs.

Neste sentido, a personalidade pode ser um elemento decisivo a ser levado em consideração em protocolos de tratamento voltados a pessoas com histórico de EEPs, visto haver possibilidade de terem se desenvolvido de forma desadaptativa e atuarem como elemento de vulnerabilidade que possa predispor essas pessoas a desenvolverem e manterem sua condição clínica.

\section{Referências}

Arata, C. M., Langhinrichsen-Rohling, J., Bowers, D., \& O’Brien, N. (2007). Differential correlates of multi-type maltreatment among urban youth. Child Abuse \& Neglect, 31(4), 393-415. doi:10.1016/j.chiabu.2006.09.006

Banducci, A. N., Hoffman, E. M., Lejuez, C. W., \& Koenen, K. C. (2014). The impact of childhood abuse on inpatient substance users: specific links with risky sex, aggression, and emotion dysregulation. Child Abuse \& Neglect, 38(5), 928-938. doi:10.1016/j.chiabu.2013.12.007

Barbosa, L. P., Quevedo, L., da Silva, G. D. G., Jansen, K., Pinheiro, R. T., Branco, J., \& da Silva, R. A. (2014). Childhood trauma and suicide risk in a sample of young individuals aged 14-35 years in southern Brazil. Child Abuse \& Neglect, 38(7), 1191-1196. doi:10.1016/j.chiabu.2014.02.008

Bernstein, D. P., Stein, J. A., Newcomb, M. D., Walker, E., Pogge, D., Ahluvalia, \& T., Zule, W. (2003). Development and validation of a brief screening version of the Childhood Trauma Questionnaire. Child Abuse \& Neglect, 27(2), 169-190. doi:10.1016/S0145-2134(02)00541-0

Carr, C. P., Martins, C. M. S., Stingel, A. M., Lemgruber, V. B., \& Juruena, M. F. (2013). The role of early life stress in adult psychiatric disorders: a systematic review according to childhood trauma subtypes. The Journal of Nervous and Mental Disease, 201(12), 1007-1020. doi:10.1097/NMD.0000000000000049

Cloninger, C. R. (1987). A systematic method for clinical description and classification of personality variants. A proposal. Archives of General Psychiatry, 44(6), 573-588. doi:10.1001/archpsyc.1987.01800180093014

Cloninger, C. R., Svrakic, D. M., \& Przybeck, T. R. (1993). A psychobiological model of temperament and character. Archives of General Psychiatry, 50(12), 975-990. doi:10.1001/archpsyc.1993.01820240059008

Cloninger, C. R., Svrakic, N. M., \& Svrakic, D. M. (1997). Role of personality self-organization in development of mental order and disorder. Development and Psychopathology, 9(04), 881-906. doi:10.1017/S095457949700148X

De Carvalho, H. W., Pereira, R., Frozi, J., Bisol, L. W., Ottoni, G. L., \& Lara, D. R. (2014). Childhood trauma is associated with maladaptive personality traits. Child Abuse \& Neglect, 44, 18-25. doi:10.1016/j.chiabu.2014. 10.013

De Clercq, B., Rettew, D. C., Althoff, R. R., \& De Bolle, M. (2012). Childhood personality types: vulnerability and adaptation over time. Journal of Child Psychology and Psychiatry, and Allied Disciplines, 53(6), 716-722. doi:10.1111/ j.1469-7610.2011.02512.x

Fassino, S., Amianto, F., Sobrero, C., \& Abbate Daga, G. (2013). Does it exist a personality core of mental illness? A systematic review on core psychobiological personality traits in mental disorders. Panminerva Medica, 55(4), 397-413. (DOI INEXISTENTE)

Finkelhor, D., Ormrod, R. K., \& Turner, H. A. (2007). Poly-victimization: A neglected component in child victimization. Child Abuse \& Neglect, 31(1), 7-26. doi:10.1016/j.chiabu.2006.06.008

Frodl, T. \& O'Keane, V. (2013). How does the brain deal with cumulative stress? A review with focus on developmental stress, HPA axis function and hippocampal structure in humans. Neurobiology of Disease, 52, 24-37. doi:10.1016/j. nbd.2012.03.012

Gadow, K. D., Sprafkin, J., \& Weiss, M. (2004). Adult Self-Report Inventory-4 manual. Stony Brook, NY: Checkmate Plus.(DOI INEXISTENTE)

Goncalves, D. M. \& Cloninger, C. R. (2010). Validation and normative studies of the Brazilian Portuguese and American versions of the Temperament and Character Inventory-Revised (TCI-R). Journal of Affective Disorders, 124(1-2), 126-133. doi:10.1016/j.jad.2009.11.007

Grassi-Oliveira, R., Cogo-Moreira, H., Salum, G. A., Brietzke, E., Viola, T. W., Manfro, G. G., \& Arteche, A. X. (2014). Childhood Trauma Questionnaire (CTQ) in Brazilian Samples of Different Age Groups: Findings from Confirmatory Factor Analysis. PLoS ONE, 9(1), e87118. doi:10.1371/journal.pone.0087118

Grassi-Oliveira, R., Stein, L. M., \& Pezzi, J. C. (2006). Translation and content validation of the Childhood Trauma Questionnaire into Portuguese language. Revista de Saúde Pública, 40(2), 249-255. doi:10.1590/S003489102006000200010

Hengartner, M. P., Cohen, L. J., Rodgers, S., Müller, M., Rössler, W., \& Ajdacic-Gross, V. (2014). Association Between Childhood Maltreatment and Normal Adult Personality Traits: Exploration of an Understudied Field. Journal of Personality Disorders, 1-14. doi:10.1521/pedi_2014_28_143 
Hovens, J. G. F. M., Giltay, E. J., Wiersma, J. E., Spinhoven, P., Penninx, B. W. J. H., \& Zitman, F. G. (2012). Impact of childhood life events and trauma on the course of depressive and anxiety disorders. Acta Psychiatrica Scandinavica, 126(3), 198-207. doi:10.1111/j.1600-0447.2011.01828.x

IBM Corporation (2011). IBM SPSS Statistics for Windows, Version 20.0. Armonk, NY: IBM Corp.

Josefsson, K., Jokela, M., Cloninger, C. R., Hintsanen, M., Salo, J., Hintsa, T., ... Keltikangas-Järvinen, L. (2013). Maturity and change in personality: developmental trends of temperament and character in adulthood. Development and Psychopathology, 25(3), 713-727. doi:10.1017/S0954579413000126

Kalmakis, K. A. \& Chandler, G. E. (2014). Adverse childhood experiences: towards a clear conceptual meaning. Journal of Advanced Nursing, 70(7), 1489-1501. doi:10.1111/jan.12329

Kessler, R. C., McLaughlin, K. A., Green, J. G., Gruber, M. J., Sampson, N. A., Zaslavsky, A. M., \&Williams, D. R. (2010). Childhood adversities and adult psychopathology in the WHO World Mental Health Surveys. The British Journal of Psychiatry: The Journal of Mental Science, 197(5), 378-385. doi:10.1192/bjp.bp.110.080499

Leverich, G. S. \& Post, R. M. (2006). Course of bipolar illness after history of childhood trauma. Lancet (London, England), 367(9516), 1040-1042. doi:10.1016/S0140-6736(06)68450-X

Li, X., Wang, Z., Hou, Y., Wang, Y., Liu, J., \& Wang, C. (2014). Effects of childhood trauma on personality in a sample of Chinese adolescents. Child Abuse \& Neglect, 38(4), 788-796. doi:10.1016/j.chiabu.2013.09.002

Lupien, S. J., McEwen, B. S., Gunnar, M. R., \& Heim, C. (2009). Effects of stress throughout the lifespan on the brain, behaviour and cognition. Nature Reviews Neuroscience, 10(6), 434-445. doi:10.1038/nrn2639

Martins, C. M. S., Tofoli, A. M. C., Baes, Cristiane V. W., \& Juruena, M. (2011). Analysis of the occurrence of early life stress in adult psychiatric patients: a systematic review. Psychology \& Neuroscience, 4(2), 219-227. doi:10.3922/j. psns.2011.2.007

Martins, C. M. S., Baes, V. W. B., C., Tofoli, S. M. de C., \& Juruena, M. F. (2014). Emotional abuse in childhood is a differential factor for the development of depression in adults. The Journal of Nervous and Mental Disease, 202(11), 774-782. doi:10.1097/NMD.0000000000000202

McCarthy, M. C. \& Lumley, M. N. (2012). Sources of emotional maltreatment and the differential development of unconditional and conditional schemas. Cognitive Behaviour Therapy, 41(4), 288-297. doi:10.1080/16506073.201 2.676669

Rademaker, A. R., Vermetten, E., Geuze, E., Muilwijk, A., \& Kleber, R. J. (2008). Self-reported early trauma as a predictor of adult personality: a study in a military sample. Journal of Clinical Psychology, 64(7), 863-875. doi:10.1002/ jclp.20495

Sahin, S., Yüksel, Ç., Güler, J., Karadayı, G., Akturan, E., Göde, E., ... Üçok, A. (2013). The history of childhood trauma among individuals with ultra high risk for psychosis is as common as among patients with first-episode schizophrenia. Early Intervention in Psychiatry, 7(4), 414-420. doi:10.1111/eip.12022

Svrakic, D. M. \& Cloninger, R. C. (2010). Epigenetic perspective on behavior development, personality, and personality disorders. Psychiatria Danubina, 22(2), 153-166. (DOI INEXISTENTE)

Svrakic, D. M., Draganic, S., Hill, K., Bayon, C., Przybeck, T. R., \& Cloninger, C. R. (2002). Temperament, character, and personality disorders: etiologic, diagnostic, treatment issues. Acta Psychiatrica Scandinavica, 106(3), 189-195. doi:10.1034/j.1600-0447.2002.02196.x

Teicher, M. H. \& Samson, J. A. (2016). Annual Research Review: Enduring neurobiological effects of childhood abuse and neglect. Journal of Child Psychology and Psychiatry, n/a-n/a. doi:10.1111/jcpp.12507

Wright, A. G. C. \& Simms, L. J. (2015). A metastructural model of mental disorders and pathological personality traits. Psychological Medicine, 45(11), 2309-2319. doi:10.1017/S0033291715000252

Zhang, T., Chow, A., Wang, L., Dai, Y., \& Xiao, Z. (2012). Role of childhood traumatic experience in personality disorders in China. Comprehensive Psychiatry, 53(6), 829-836. doi:10.1016/j.comppsych.2011.10.004

Dados dos autores:

Ismael Ferreira da Costa - Mestre, Universidade Federal da Paraíba.

Maria Paula Bento Tomaz - Psicóloga, Universidade Federal da Paraíba.

Mirelly Gomes de Araújo - Psicóloga, Universidade Federal da Paraíba.

Natany de Souza Batista Medeiros - Mestre, Universidade Federal do Rio Grande do Norte.

Melyssa Kellyane Cavalcanti Galdino - Doutora, Universidade Federal da Paraíba.

Endereço para correspondência:

Ismael Ferreira da Costa

Universidade Federal da Paraíba (UFPB), Campus I - Lot. Cidade Universitária

58033-455, João Pessoa, PB, Brasil

E-mail: ismaelferr@gmail.com

Recebido em: 21/12/2017.

Aceito em: 21/11/2018.

Publicado em: 09/05/2019. 